\title{
Hybrid Intelligent Warning System for Boiler tube Leak Trips
}

\author{
Deshvin Singh ${ }^{1}$, Firas B. Ismail ${ }^{1 *}$, and Mohammad Shakir Nasif ${ }^{2}$ \\ ${ }^{1}$ Power Generation, Institute of Power Engineering (IPE), Universiti Tenaga Nasional, 43000 Kajang, \\ Selangor, Malaysia \\ ${ }^{2}$ Mechanical Engineering Department, Universiti Teknologi Petronas, 32610 Tronoh, Malaysia
}

\begin{abstract}
Repeated boiler tube leak trips in coal fired power plants can increase operating cost significantly. An early detection and diagnosis of boiler trips is essential for continuous safe operations in the plant. In this study two artificial intelligent monitoring systems specialized in boiler tube leak trips have been proposed. The first intelligent warning system (IWS-1) represents the use of pure artificial neural network system whereas the second intelligent warning system (IWS-2) represents merging of genetic algorithms and artificial neural networks as a hybrid intelligent system. The Extreme Learning Machine (ELM) methodology was also adopted in IWS1 and compared with traditional training algorithms. Genetic algorithm (GA) was adopted in IWS-2 to optimize the ANN topology and the boiler parameters. An integrated data preparation framework was established for 3 real cases of boiler tube leak trip based on a thermal power plant in Malaysia. Both the IWSs were developed using MATLAB coding for training and validation. The hybrid IWS-2 performed better than IWS-1.The developed system was validated to be able to predict trips before the plant monitoring system. The proposed artificial intelligent system could be adopted as a reliable monitoring system of the thermal power plant boilers.
\end{abstract}

\section{Introduction}

Steam boilers represent one of the main component in the power plant. Boiler tubes continuously pass fluids, withstanding extremely high temperatures and pressures of the steam generator [1]. This eventually leads to leakages in boiler tubes. Malfunction of boiler tube causes frequent boiler trips which eventually increase the operating costs of a plant [2]. Conventional detection methods of detecting tube leaks by measuring the make-up water or inspecting is ineffective as it gives information on the existing tube leak only when serious damage is already done. Consequently prediction of boiler tube leak trips is crucial to maintain normal and safe operational conditions of the plant [3]. Benefits of an early detection of boiler tube leak trip are: to increase operating profit by reducing repair costs and secondary damage, increase safety of the plant, increase availability and tube life, and avoid unplanned outages [4]. Prediction can be achieved by developing intelligent warning system which monitors the operating conditions. Using information provided by measurements from

*Corresponding author: firas@uniten.edu.my 
several sensors and actuators that are abundantly stored throughout operations, the intelligent warning system is able to predict a failure [5].

One of the most commonly used intelligent systems for early fault detection is the Artificial Neural Network. A study on ANN had adopted the methodology of feed forward backpropagation to diagnosis a steam boiler based on superheater monitoring, in which the minimisation algorithms were applied [6]. ANN as a stand-alone system is effective however there are several other intelligent systems that have been applied together with ANN for early fault detection. Genetic algorithm is an optimization tool which reduces the possibilities of getting into local minimum in the process of finding an optimum solution [7]. Momeni et al. have applied hybrid genetic algorithm-based ANN for the prediction of pile bearing capacity. From this study it was concluded that using the optimum Genetic Algorithm (GA) parameters, the hybrid model outperformed the conventional ANN model in the prediction process [8].

In all proposed solutions, there has not been any intelligent monitoring system established particularly for boiler tube leak trip in thermal power plants. Several researches use mathematically simulated data instead of real plant data for training and validation, which is inaccurate for decision making. It can be noted from previous applications that the raw data are used directly for the training process. However the data are not always complete and the quality is limited. Therefore a data preparation framework is important for the complex data analysis. Previously, trial and error approach has been applied for the selection of best NN topology combination and the optimization of the boiler operation parameters. The selection of suitable boiler operation variables and the design of the optimal neural network topology can be achieved using additional artificial intelligent system technique such as GA. Therefore the objective of this research is to develop two Intelligent Warning Systems (IWSs) for boiler trip prediction by adopting pure artificial neural network system and hybrid intelligent system. IWS-1 represents the use of the pure artificial neural network system for boiler trip prediction. The ELM training algorithm was also adopted for IWS-1 and the results were compared with the traditional training algorithms. IWS-2 involves the hybrid intelligent system using the application of artificial neural networks with genetic algorithms. In order to ease the selection of suitable boiler operation variables and to achieve the optimal neural network topology, encoding and optimization process is done using genetic algorithms.

\section{Description of power plant and trip cases}

This study was focused on three real cases of boiler tube leak trip that had occurred in a thermal power plant in Malaysia. This particular power plant has six operational units with capacity of generating up to $2420 \mathrm{MWe}$. The units have triple fuel firing capability using gas, oil and coal. The steam generating capacity for the unit 1 is 169 bar whereas the steam outlet has a temperature of $560{ }^{\circ} \mathrm{C}$. The tubes used in the boilers are made up of stainless alloy heater tubes of various grades. The ratio of unplanned outages against total annual hours was at $12.5 \%$ in the year 2005 . Over the years the number of unplanned outages have declined significantly for all the units, however the operating company is targeting for lower number of planned outages. One of the main reason has been boiler tube leak trips which eventually causes shutdown and outage.

The scope of this work focusses on the working fluid of the boiler components including drum, superheater, boiler feed pump and water tube. These components are chosen based on the frequency of the trip occurrence within a set period of time. The real cases of boiler tube leak trips that had occurred unit 1 have been considered for this study. The first case was a boiler tube leak trip caused due to sudden overheating of boiler tubes. The second trip was caused by steam temperature fluctuation whereas the third case was due to extremely high superheater steam temperature. 


\section{Data acquisition and preparation}

The on-line plant control system consists of 1250 observations of actuators and sensor signals which are identified as the boiler process observations. This large number of observation is reduced to 67 based on the work scope which focuses on the diagnosis of working fluid components only in the boiler. Since many of the observations were measured by multiple sensors, eventually only 17 influential operation boiler variables were identified to be used as input. The enormously large data collected from the power plant containing thousands of values is filtered and passed through three important steps. They are visual cleaning of data, missing data treatment and data normalisation. Data normalisation was used as a data transformation technique to scale the data between 0 and 1 ( 0 represents running mode and 1 represents faulty). The Min-Max data normalisation was applied. For each variable, the behavioural analysis was done based on its time interval to reach high alarm indicator at " 1 ".

\section{Design and implementation of IWSs}

In this study the feed-forward methodology was adopted using the back-propagation neural network. Activation functions are applied to the weighted sum of its input. The main NN topologies are training algorithms, learning rate, momentum coefficient, activation functions, number of hidden layers and the number of neurons in each hidden layers. In this study up to 2 hidden layers have been used. The performances of these systems are indicated by Root Mean Square Error (RMSE) where output error between the actual output and the expected output is evaluated. It is noted that lower error value indicates better performance. Based on the best performance of the IWS, training and validation is done. The 17 variables presented before are used as inputs in the IWS and the outputs are either ' 0 ' or ' 1 '. The ANN training is done in two phases: the preliminary training phase and the basic training phase.

\subsection{Design Implementation of IWS-1}

The main objective of this preliminary training is to determine with the optimal $\mathrm{NN}$ topology combination. The IWS was input with faulty data set which makes up $60 \%$ of the data set. The network input includes all 17 operation variables. The optimal topology combination obtained from the preliminary training results is used for the basic training. Real boiler data set were presented as basic training sets for the IWS-I. Based on the proposed system performance indicator, the basic training results were analysed. The calculated RMSE values of the optimal NN topology combination is compared with the RMSE value of the preliminary training process. The aim of this process is to get smaller or equal calculated RMSE values than the preliminary training process. In the validation and testing process the last $40 \%$ of the data set was used as a validation real data set. Applying the optimal NN topology combination obtained from the preliminary training process, validation of real data set was used to determine the rapidness of the proposed IWS in predicting the specific trip. The performance of the system during normal boiler operation is also validated.

\subsection{Design Implementation of IWS-2}

In the development of IWS-2, GA is used for automated design and NN parameter optimisation. In this work, IWS-2 combined ANN with GA in order to determine the best ANN structure and to optimise the ANN input parameters. The NN topologies were encoded together with the NN training parameters into the genes of the GA using Weak Specification Representation (WSR). The proposed GA individual has genotypes which represent every 
phenotype of ANN topologies together with training parameters. Binary representations were established for activation function, neuron number and input parameters. At first, an initial population is generated randomly in which the parents are selected. Crossover and mutation operators are applied on the parents to generate new generation on population. In this study, random initialisation is adopted for population initialisation because heuristic initialisation can result in the population having similar solutions. The population size is set by the user in which a bigger population will consume longer computational time. Fitness function is a function which determines how effective the solution is with respect to the considered problem. In this study the fitness function was represented by the RMSE value obtained from the ANN training. The selection operator used in this study is the fitness based selection. Using this selection operator, the new offspring replaces the least fit individuals in the population. The number of generation is set to be the termination condition for GA. GA is run for maximum number of iterations until there is no improvement in the population.

\section{Results analysis}

The performance of each system is analysed and discussed in this section based on the best $\mathrm{NN}$ topology combination together with the most influential boiler variables. In the training process of IWS-1 for case 1, the resilient back-propagation algorithm performed best among the traditional algorithms. However ELM was able to produce better results in terms of RMSE value. It can be concluded that for all cases, the minimum RMSE values were obtained using ELM. It can be concluded that the ELM algorithm performed better than the traditional ANN algorithm. Therefore the ELM algorithm is applied for the next step which is validation. Using the optimal results achieved from IWS-2, the validation for each case was done with the same validation data sets used for IWS-1 validation. Similar approach of IWS-1 was adopted in indicating the fault in IWS-2. Figure 1 represents IWS-2 output results for all cases. In case 1, the trip was introduced at the 15220th interval. IWS-2 was able to detect the fault at the 15210th interval with an output of 0.7 . This output is considered above average warning indication. For case 2, the total data sampling interval is at the point of 15063th minute before the shutdown. The introduction of fault in the operation occurred at the $15051 \mathrm{st}$ interval. The intelligent system was able to predict the fault within the 15040th interval. The fault indication is considered a strong alarm warning indication (close to one) with system output of 0.91 . In case 3 , the total data sampling interval is at the point of 16600 th minute before the shutdown. The introduction of fault in the operation occurred at the 16570th interval. The intelligent system was able to predict the fault within the 16550th interval. The fault indication is considered a very strong alarm warning indication (close to one) with system output of 0.93 .

\subsection{Comparison between IWS-1 and IWS-2 performance results}

Both the systems were able to detect specific boiler tube leak trips based on the trip cases with varied performances. For IWS-1, it was established that the ELM training algorithm outperformed the conventional ANN training algorithms. Therefore ELM was adopted in the hybrid system IWS-2. Table 1 summarises the results of IWS-1 and IWS-2 for all cases. In case 1, IWS-1 detected the trip 9 minutes earlier than IWS-2. Similar comparison can be done for case 2 and 3 in which the detection time of IWS-1 outperformed IWS-2. However in all the trip cases, IWS-2 resulted in lower RMSE values compared to IWS-1. This indicates that IWS-2 performed better than IWS-1 in all cases. The output value is referred to categorise the alarm warning level based on the thresholds set. The closer the output value is to ' 1 ', the stronger the alarm warning indication. Therefore a direct comparison of results using RMSE 
or detection time cannot determine the better system. Despite being able to achieve the objective of detecting the boiler tube leak trips, IWS-1 required longer time in developing the structure of ANN and selecting the input variables. This is due to the trial and error approach which is not efficient when it comes to applying the same IWS method for other boiler units or trip cases. Optimisation of the effective variables and NN architecture is essential, considering the large problem space of topology combinations and input variables available. IWS-2 merged optimisation techniques with $\mathrm{NN}$ techniques in a hybrid system, to select the best structure combination and input variables. This way, the trial and error process can be avoided all together saving time in developing the IWS. Using IWS-2 the structure development and input variables selection was simplified, thus making the application of the system for other boiler units and trip cases less tedious.

a)

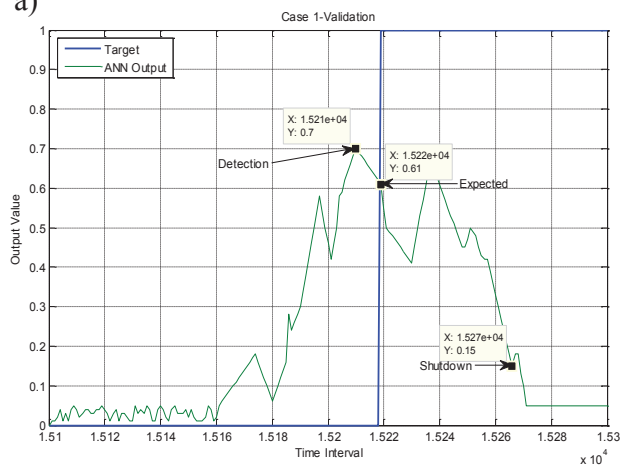

b)

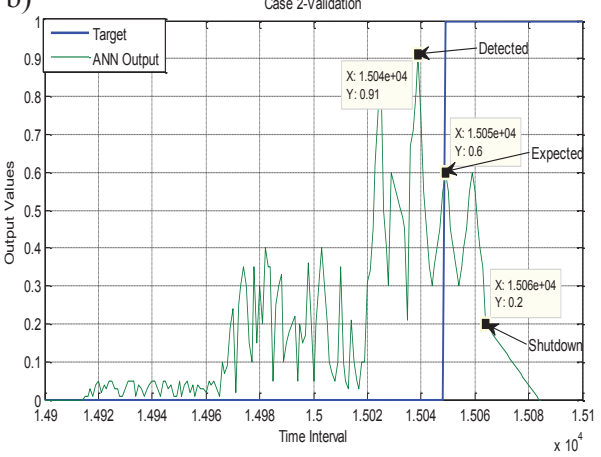

c)

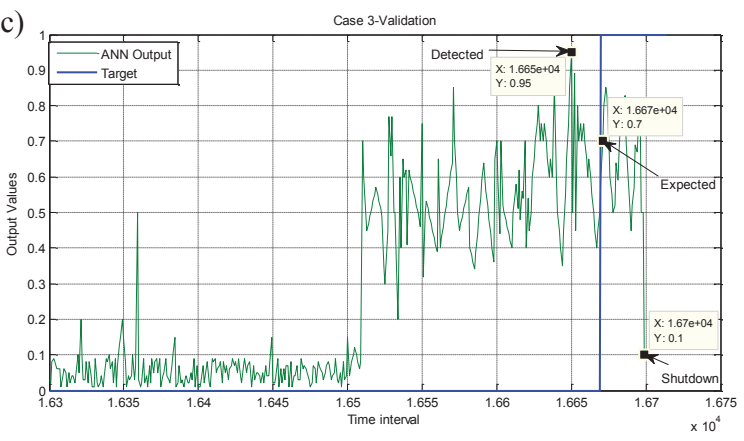

Fig. 1. IWS-2 output for: a) Case 1; b) Case 2; c) Case 3

Table 1. Summary of IWS-1 and IWS-2 results for all cases.

\begin{tabular}{|l|c|c|c|c|c|c|}
\hline Trip cases & \multicolumn{2}{|c|}{ Case 1 } & \multicolumn{2}{c|}{ Case 2 } & \multicolumn{2}{c|}{ Case 3 } \\
\hline Systems & IWS-1 & IWS-2 & IWS-1 & IWS-2 & IWS-1 & IWS-2 \\
\hline Architecture & $9 \mathrm{HL} 1$ & 7HL1 & $5 \mathrm{HL} 1$ & $7 \mathrm{HL} 1$ & $8 \mathrm{HL} 1$ & $6 \mathrm{HL} 1$ \\
\hline Activation function & L+P & L+P & L+L & L+P & L $+\mathrm{P}$ & L+L \\
\hline RMSE & 0.1421 & 0.1419 & 0.1618 & 0.1312 & 0.0931 & 0.0701 \\
\hline Detection & 19 & 10 & 22 & 11 & 30 & 20 \\
\hline Output & 0.75 & 0.7 & 0.9 & 0.91 & 0.95 & 0.93 \\
\hline Trip probability & \multirow{2}{*}{ High } & \multirow{2}{*}{ High } & \multirow{2}{*}{ High } & \multirow{2}{*}{ Very high } & \multirow{2}{*}{ Very high } & \multirow{2}{*}{ Very high } \\
\hline
\end{tabular}




\section{Conclusion}

Early detection of boiler tube leak trip was achieved for all three cases. Two IWS were developed using pure ANN and combination of ANN with GA. Using real faulty data, the proposed systems were validated. An integrated data preparation framework has been proposed to process the large amount of data. The single layer feed-forward neural network with ELM training algorithm performed better than the traditional training algorithms. The best ANN topology combination for each trip case was determined. IWS-1 was able to detect the trips earlier than IWS-2. However taking into consideration the optimization capability of GA, IWS-2 was selected as the reliable system for this boiler unit. The optimization of ANN topology and input boiler parameters has made the development of IWS simpler and faster. Hybrid intelligent warning system can be a reliable system for warning and analyzing faults in power plants. Boiler tube leak trips were successfully detected earlier by the IWS in order to allow the plant operator to take appropriate action in maintaining continuous operation of boilers.

Acknowledgement to Ministry of Higher Education Malaysia (MOHE) for sponsoring this study in Universiti Tenaga Nasional through the MyBrain15 Scholarship Program.

\section{References}

1. P.K. Nag, Power Plant Engineering (Tata McGreaw-Hill, 2002).

2. T. Zhong, A.T. Alouani, R.A. Smoak, System Theory (1997).

3. Jianping Ma and Jin Jiang, Progress in Nuclear Energy (2011) pp.255-266.

4. D. Flynn, Thermal power plant simulation and control (The Institution of Electrical Engineers, 2003).

5. L. Fortuna, S. Graziani, A. Rizzo, Xibilia M.G., Advances in Industrial Control (2007).

6. B. Firas and H. Hussain, Journal of Applied Sciences (2011).

7. T. Jingwen and G. Meijuan, "Network intrusion detection method based on high speed and precise genetic algorithm neural network", Conference on Network Security, Wireless Communications and Trusted Computing (2009).

8. E. Momeni, R. Nazir, D. Jahed and H. Maizir, Measurement, 57 (2014) pp.122-131. 